\title{
Poster Session 4
}

\section{4-1 [\#3031]}

\section{Cross-coupled stimulus during artificial gravity:} Asymmetric tumbling sensation response

J. Mateus ${ }^{\mathrm{a}}$, A.N. Hearn ${ }^{\mathrm{b}}$, J. Cañizales ${ }^{\mathrm{a}}$ and L.R. Young ${ }^{\mathrm{a}}$

${ }^{a}$ Man-Vehicle Laboratory, Department of Aeronautics and Astronautics, Massachusetts Institute of Technology, 77 Mass. Ave., Room 37-219, Cambridge, MA 02139,USA.E-mail: jm@mit.edu

${ }^{\mathrm{b}}$ School of Electrical Electronic and Mechanical Engineering, National University of Ireland, Dublin, Ireland

Artificial gravity (AG) through centrifugation is a promising countermeasure for long-duration spaceflight physiological deconditioning. Vestibular adaptation to $A G$ is necessary in order to mitigate the negative side effects associated with the cross-coupled stimulus (CCS) that results from performing head turns out of the plane of centrifuge rotation. Previous research has investigated many of the factors that contribute to the CCS such as head turn velocity, centrifuge velocity, and magnitude of head turn rotation, among others. Most of these can be understood by an analysis of the physics of the CCS. However, we have consistently noted an asymmetry between clockwise (CW) and counter-clockwise (CCW) yaw-axis head turns. This asymmetric response is not readily explained by the physics of the CCS, and it has also not been adequately explained by any of the existing models of the vestibular system. We have characterized the asymmetry of the CCS through three experiments in which we provide yaw head turns during $\mathrm{AG}$ with: 1) $\mathrm{CW}$ supine, 2) $\mathrm{CCW}$ supine, and 3) $\mathrm{CW}$ prone centrifugation. The data indicate that head turns resulting in a subjective tumbling sensation of rotating away from the horizontal centrifuge bed always produce a more intense tumbling sensation than head turns that lead to a sensation of rotating into the bed. We propose that the perceived dan- ger of the CCS-induced tumbling sensation movement modulates the subjective tumbling intensity responses.

Acknowledgement: This work was supported by the National Space Biomedical Research Institute NASA NCC 9-58.

\section{4-2 [\#3035]}

Effects of labyrinthectomy on rat soleus muscles properties and lumbar motoneurons

\author{
M. Falempin and F. Picquet \\ University Lille Nord de France, F-59000 Lille, France
}

The aims of this study were to determine whether the suppression of the vestibular inputs 1) could have effects on the soleus muscle properties similar to the modifications observed after an episode of artificial microgravity and 2) could affect the morphology and the excitability of the motoneuron pool which innervate the soleus.

The inner ear lesion was obtained by surgical labyrinthectomy. Male Wistar rats were used and divided in 2 groups: control (CONT, $n=21$ ) and bilateral labyrinthectomised (BL, $n=14)$. Mechanical, histochemical, electrophoretic, electrophysiological parameters and soleus electromyographic (EMG) activity were measured 17 days after the surgical operation.

Our results showed that rat soleus of the BL group were not atrophied when compared to CONT group. The ratio of peak titanic tension relative to the muscle wet weight was not changed. However, the kinetic parameters and the phenotypical profile determined following $\mathrm{MHC}$ isoforms revealed that the BL soleus muscle evolved from a slow towards a slower type. This has been correlated to a more tonic EMG activity pattern. The soma sizes of the soleus motoneuron pool of the BL group were shifted towards smaller size classes. A decrease in the monosynaptic reflex thresh- 
old and an increase in the monosynaptic reflex latency were observed in the BL group.

Therefore, our data demonstrated that the soleus muscle transformations observed after simulated microgravity cannot be the result of a vestibular silence. However the loss of vestibular information led to modifications of the motoneuron properties.

Acknowledgement: Supported by grants from the French "Centre National d'Etudes Spatiales"- CNES, 8411.

\section{4-3 [\#3048]}

Denoising of impedance cardiography data from returning astronauts using the Ensemble Empirical Mode Decomposition method: Preliminary results from the ESA SPIN experiment

P.-F. Migeotte ${ }^{\mathrm{a}}$, T. Peeters ${ }^{\mathrm{a}}$, A. Diedrich ${ }^{\mathrm{c}}$, X. Neyt ${ }^{\mathrm{a}}$, N. Pattyn $^{\mathrm{a}, \mathrm{b}}$ and F. Wuyts ${ }^{\mathrm{d}}$

${ }^{a}$ Royal Military Academy, Electrical Engineering Department, Signal and Image Centre, Brussels, Belgium. E-mail: Pierre-francois.migeotte@elec.rma.ac.be ${ }^{\mathrm{b}}$ Vrije Universiteit Brussel, Department of Biological Psychology, Brussels, Belgium

${ }^{\mathrm{S}}$ School of Engineering, Vanderbilt University, Nashville, TN, USA

${ }^{\mathrm{d}}$ Antwerp University Research Centre for Equilibrium and Aerospace (AUREA), University of Antwerp, Antwerp, Belgium

Impedance cardiography is a robust and well documented non-invasive method for measuring heart stroke volume. This technique is combined with the recording of impedance in 4 body segments to measure the fluid shifts during a standardized-tilt test protocol performed on 4 astronauts returning from long duration missions ( $\sim 6$ months) to the ISS. This protocol is part of the ESA SPIN study on otolith and autonomic deconditioning after spaceflight and is performed after rotation of the subjects on the VVIS centrifuge.

In returning astronauts the data acquisition is often altered by noise from physical as well as physiological origin. The Ensemble Empirical Mode Decomposition (EEMD) is a novel technique ideal for non-linear and non-stationary signals [1] which adaptively decomposes a signal into Intrinsic Mode Functions (IMFs) [2].

The performance of the EEMD denoising algorithm is compared with a Wavelet coefficient thresholding algorithm. Artificial model wave shapes were construct- ed and white noise was added to test both algorithms. In most cases the EEMD technique outperformed the Wavelet thresholding method.

EEMD was applied to the recordings (3 pre-flight BDC \& 3 post-flight $(\mathrm{R}+1, \mathrm{R}+4, \mathrm{R}+9))$ of the SPIN study. Our results show that EEMD allowed a denoising method tailored to our problem. Moreover the IMF that showed to be consistently correlated with the respiratory signal was extracted as a surrogate respiratory signal. Impedance in the 4 segments of the body (thorax, abdomen, thigh and calf) were calibrated and show that after space flight the fluid shift is more pronounced compared to the pre-flight baseline.

Acknowledgement: This research was supported by the Belgian Federal Science Policy Office (BELSPO) via the European Space Agency PRODEX program.

\section{References}

[1] Z. Wu and N.E. Huang, Ensemble Empirical Mode Decomposition: a noise-assisted data analysis method, Advances in Adaptive Data Analysis 1(1) (2009), 1-41.

[2] N.E. Huang, Z. Shen, S.R. Long, M.C. Wu, H.H. Shih, Q. Zheng, N.C. Yen, C.C. Tung and H.H. Liu, 1998, The empirical mode decomposition and the Hilbert spectrum for nonlinear and non-stationary time series analysis, Proceedings: Mathematical, Physical and Engineering Sciences 454 (1971), 903-995.

\section{4-4 [\#3027]}

Adaptation to coriolis-inducing head movements in a sustained-G high performance flight simulator

M.C. Newman ${ }^{\text {a }}$, G.W. McCarthy ${ }^{\mathrm{a}}$, P.W. Comtois ${ }^{\mathrm{a}}$, S.T. Glaser $^{\mathrm{b}}$ and $\mathrm{F}$. Bonato ${ }^{\mathrm{c}}$

${ }^{a}$ National Aerospace Training and Research Center, Southampton, PA 18966, USA. E-mail: mnewman@ etcusa.com

${ }^{\mathrm{b}}$ The Defiant Company, Rosamond, CA 93560, USA

${ }^{\mathrm{c}}$ Human Perception and Performance Lab, Saint Peter's College, Jersey City, NJ 07306, USA

The goal of the present experiment is to investigate and quantify cognitive and physiological adaptation to head movements made in a sustained $G$ high performance flight simulator. Sustained G simulators combine long arm centrifugation with high fidelity, gimbaled, flyable cockpit modules to mimic the physiological stresses and $\mathrm{G}$ forces experienced during actual tactical flight. In order to properly reproduce these forces, 
high rotational rates up to $250 \%$ sec of the centrifuge arm are required. A head movement made about an axis other than that of the planetary arm will produce an instantaneous stimulus to the semi circular canals, about a third axis, that can often be disorienting and nauseogenic. The resultant perceptual illusion of tiling and tumbling is referred to as the Coriolis or vestibular Cross-Coupling Effect. Because tactical flying is rarely eyes-forward, immobile, head-fixed flying, it is desirable in a tactical flight simulator to minimize these unwanted perceptual motion artifacts within the full range of head and neck motion. This study seeks to examine the effect repeated Coriolis- inducing head movements have on the intensity and nauseogenicity of the resultant perceptual response. Ten acrobatic and/or fighter pilots will perform a 4 day adaptation protocol. Subjects will make head movements to projected and/or fixed visual targets within the gondola cockpit (Range: Left-Right $\pm 90^{\circ} \mathrm{Up}$-Down $\pm 45^{\circ}$ ) and will report their motion sickness rating and tumbling intensity as symptoms develop. Residual adaptation retention levels will be assessed at one and two weeks following the final exposure. With a better understanding of the rate, degree, and retention level of possible adaptation, a training program will be developed to minimize initial symptoms, extend training durations and optimize the benefits of sustained $\mathrm{G}$ flight simulation. Preliminary results, if available, will be presented and discussed.

\section{4-5 [\#3001]}

\section{Astronaut spatial orientation perceptions during simulated lunar landing}

T.K. Clark ${ }^{\mathrm{a}}$, L.R. Young ${ }^{\mathrm{a}}$, A.J. Stimpson ${ }^{\mathrm{a}}$, K.R. Dudab, C.M. Oman ${ }^{\mathrm{a}}$ and A. Natapoff ${ }^{\mathrm{a}}$

${ }^{a}$ MIT, 77 Massachusetts Avenue 37-219, Cambridge, MA02139,USA.E-mail: torin@mit.edu

${ }^{\mathrm{b}}$ The Charles Stark Draper Laboratory, Inc., 555 Technology Square, Cambridge, MA 02139, USA. E-mail: kduda@draper.com

Lunar landing requires the identification and selection of a suitable landing zone and a safe and precise descent to the surface. In crewed landings, astronauts are expected to interact with automated systems to accomplish those objectives through supervisory and possibly manual control. Astronauts must maintain accurate perceptions of vehicle orientation and velocity while maintaining terrain and situational awareness. A simulation was run on a physiologically-based, numerical model of visual-vestibular interaction using vehicle motions during landing trajectories to predict the astronaut's perceptions of vehicle orientation. An experiment was performed in which subjects reported their perceptions of vehicle attitude and horizontal velocity during motions typical of lunar landing simulated on the NASA Ames Research Center Vertical Motion Simulator (VMS). During those motions, we studied subjects' perceptions resulting from three different sets of orientation cues: Vestibular only (VO), visual-out-thewindow view (VOTW), and instrument display panel (IDP). Both the numerical simulation and experimental results found a somatogravic illusion in the VO (blindfolded) case: subjects reported upright vehicle orientation even when the vehicle model imposed a significant tilt. The model predicts that having a view of the lunar terrain will improve the perception of vehicle orientation, but the experimental results did not show that predicted improvement. The somatogravic illusion was suppressed when the subjects had access to the IDP. Finally, the experiment found misperceptions of horizontal velocity in the VO and VOTW cases. These misperceptions of vehicle attitude and velocity are likely to degrade astronaut control and may impact landing performance and safety.

Acknowledgement: This work was supported by the National Space Biomedical Research Institute through NASA NCC9-58-11 Projects SA01604 and SA01302.

\section{4-6 [\#3042]}

\section{Effect of dynamic otolith stimulation in pitch tilt in healthy human subjects}

\author{
G. Bertolini ${ }^{\mathrm{a}, \mathrm{b}}$, S. Ramat ${ }^{\mathrm{b}}$, D. Straumann ${ }^{\mathrm{a}}$, A. Wicki $^{\mathrm{a}}$ \\ and A. Palla ${ }^{\mathrm{a}}$ \\ a Department of Neurology, Zurich University Hospital, \\ Zurich, Switzerland. E-mail: bertoweb@gmail.com \\ ${ }^{\mathrm{b}}$ Department of Computer Science, University of Pavia, \\ Pavia, Italy
}

Altered-gravity environment challenges our selfmotion perception. The velocity-storage (VS) that determines the rotational vestibulo-ocular reflex (rVOR) behavior, also critically contributes to self-motion perception [1]. During earth-horizontal axis rotation (EHR) VS processing is influenced by otolith signals. By exposing healthy subjects (hS) to 'natural' and altered forward pitch EHR, we aimed to elucidate if the 
effect of dynamic otolith stimulation $\left(\mathrm{OTO}_{d y n}\right)$ on the VS can explain both, the rVOR and self-motion perception behavior. rVOR was recorded with dual-search coils during per- ('natural' $\mathrm{OTO}_{d y n}$ ) and post-rotatory (altered $\mathrm{OTO}_{d y n}$ ) constant-velocity pitch EHR. Pitchtilt perception was assessed by asking hS, which of two consecutive motion stimuli was perceived as larger tilt. One stimulus (reference-stimulus) consisted of a constant-velocity pitch-tilt, the other of either: (1) a smaller/larger pitch-tilt ('natural' OTO ${ }_{d y n}$ ), (2) a smaller/larger pitch-tilt combined with a surge translation providing $\mathrm{OTO}_{d y n}$ equal to the reference-stimulus (altered $\left.\mathrm{OTO}_{d y n}\right)$. Per-rotatory slow-phase eye-velocity $(7.8 \pm 2.1 \mathrm{~s})$ decayed significantly slower than postrotatory slow-phase eye-velocity $(3.6 \pm 0.7 \mathrm{~s})[2]$. During pitch-tilts, hS recognized larger tilts in $83.5 \pm 1$ $5.8 \%$ of trials in paradigm (1) compared to $70.3 \pm$ $21.7 \%$ in paradigm $(2)(p<0.001)$. This demonstrates that the proper activation of VS by 'natural' gravity-dependent OTO $_{d y n}$ has a striking role in both, the rVOR and self-motion perception. We speculate that in altered-gravity environment, $\mathrm{OTO}_{d y n}$ that differs from the one expected on Earth may cause incorrect VS processing of low-frequency components of rotational stimuli, possibly affecting pitch-tilt assessment.

\section{References}

[1] Bertolini et al., J Neurophysiol, 2010.

[2] Bertolini, Ramat, Exp Brain Res, in press.

\section{4-7 [\#3057]}

Space flight experiments reveal gravity-related critical periods in vestibular and tail development of an amphibian (Xenopus laevis)

\section{E. Horn}

Institute of Neurobiology, Ulm University, AlbertEinstein-Allee 11, 89081 Ulm, Germany. E-mail: eberhard.horn@uni-ulm.de

Development of sensory systems is characterized by periods of life (critical period) during that animals are susceptible to environmental modifications, in particular to sensory deprivation. In 1993 we started a project to find out whether space weightlessness at glevels between $10^{-3} \mathrm{~g}$ to $10^{-5} \mathrm{~g}$ affects the morphological development of Xenopus laevis and, in partic- ular, whether its static roll-induced vestibuloocular reflex (rVOR) has a g-related critical period. STATEX on Spacelab mission STS-55 (1993) was the first experiment of this series, followed by experiments TADPOLE on STS-84 (1997) and AQUARIUS-XENOPUS on Soyuz TM33/TM32 (2001). Experiment XENOPUS on Soyuz TMA13/TMA12 (2008) was the 4th and last experiment. The obtained results obtained from these 9 to 12 days lasting missions allow us to define g-related critical periods in rVOR and tail development. (1) Concerning rVOR development, affected stages were close to a 2-days lasting period of life when the rVOR appeared for the first time, i.e., probably when synaptic contacts in the underlying vestibular pathways are formed. (2) Tail development was studied by means of tail lordosis that is an upward bend of the tail typical for tadpoles during orbital flights. There is evidence for a g-related sensitivity of tail development during the period of life ranging from the tail bud to the forelimb bud stages.

Acknowledgement: Supported by DLR, grant 50 WB0630.

\section{4-8 [\#3006]}

\section{Reduction in Dynamic Visual Acuity reveals gaze control changes following spaceflight}

B.T. Peters ${ }^{\mathrm{a}}$, R.A. Brady ${ }^{\mathrm{a}}$, C.A. Miller ${ }^{\mathrm{a}}$, E.L. Lawrence $^{\mathrm{a}}$, A.P. Mulavara ${ }^{\mathrm{b}}$ and J.J. Bloomberg ${ }^{\mathrm{c}}$

${ }^{\mathrm{a}}$ Wyle Integrated Science and Engineering Group, Houston, TX, USA

${ }^{\mathrm{b}}$ Universities Space Research Association Division of Space Life Sciences, Houston, TX, USA

${ }^{\mathrm{c}}$ NASA Johnson Space Center, Houston, TX, USA

Introduction: Exposure to microgravity causes adaptive changes in eye-head coordination that can lead to altered gaze control. This could affect postflight visual acuity during head and body motion. The goal of this study was to characterize changes in dynamic visual acuity after long-duration spaceflight.

Methods: Dynamic Visual Acuity (DVA) data from 14 astro/cosmonauts were collected after long-duration ( $\sim 6$ months) spaceflight. The difference in acuity between seated and walking conditions provided a metric of change in the subjects' ability to maintain gaze fixation during self-motion. In each condition, a psychophysical threshold detection algorithm was used to display Landolt ring optotypes at a size that was near 
each subject's acuity threshold. Verbal responses regarding the orientation of the gap were recorded as the optotypes appeared sequentially on a computer display 4 meters away. During the walking trials, subjects walked at $6.4 \mathrm{~km} / \mathrm{h}$ on a motorized treadmill.

Results: A decrement in mean postflight DVA was found, with mean values returning to baseline within 1 week. The population mean showed a consistent improvement in DVA performance, but it was accompanied by high variability. A closer examination of the individual subject's recovery curves revealed that many did not follow a pattern of continuous improvement with each passing day. When adjusted on the basis of previous long-duration flight experience, the population mean shows a "bounce" in the re-adaptation curve.

Conclusion: Gaze control during self-motion is altered following long-duration spaceflight and changes in postflight DVA performance indicate that vestibular re-adaptation may be more complex than a gradual return to normal.

\section{4-9 [\#3012]}

\section{Lateral tilt illusion caused by idiopathic utricular dysfunction. Is it detectable by ocular VEMP?}

T. Murofushi, H. Nakahara, Y. Tsuda and E. Yoshimura Department of Otolaryngology, Teikyo University School of Medicine, Mizonokkuchi Hospital, 3-8-3 Mizonokuchi Takatsu-ku Kawasaki 213-8507, Japan. E-mail: toshi-tky@umin.ac.jp

The vestibular labyrinth is composed of two otolith organs and 3 semicircular canals. The otolith organs, utricle and saccule, work as sensors of linear acceleration. From the viewpoint of polarity, the utricular macula seems to be sensitive to lateral tilts. Therefore, its dysfunction could lead to lateral tilt illusion. Vestibular evoked myogenic potentials around the eye (ocular VEMP, oVEMP) have been recognized as a clinical test of utricular function. Herein, in order to confirm validity of oVEMP as a test of utricular function, we studied oVEMP in patients presented with idiopathic lateral tilt illusion. Patients presented with episodes with idiopathic lateral tilt illusion were enrolled. It was the inclusion criterion for subjects to have episodic lateral tilt sensation including sensations pushed or pulled laterally. Subjects with the following signs or symptoms were excluded. 1. Episodic rotatory verti- go, or canal dysfunction detected by caloric tests (canal paresis $>20 \%$ or bilateral canal dysfunction, maximum slow phase eye velocity $<10 \mathrm{deg} / \mathrm{sec}$ bilaterally). 2. Episodes of loss of consciousness or black out sensation). 3. Any symptoms or signs of central nervous system dysfunctions or proprioceptive dysfunctions. 4. Definite diagnoses of known diseases which cause disequilibrium (e.g. Meniere's disease). For patients who passed the above-mentioned inclusion and exclusion criteria, oVEMP and cVEMP were recorded using $500 \mathrm{~Hz}$ air-conducted tone bursts.

Majority of subjects showed unilateral or bilateral absence of oVEMP but preserved cVEMP. These findings suggested that lateral tilt illusion could be caused by idiopathic utricular dysfunction and such dysfunction is detectable using oVEMP.

\section{4-10 [\#3018]}

\section{Novel tool development to assess vestibular health}

S.L. Kukreja ${ }^{\mathrm{a}}$ and R.D. Boyle ${ }^{\mathrm{b}}$

${ }^{a}$ NASA, Dryden Flight Research Center, Edwards, CA 93523,USA.E-mail: Sunil.L.Kukreja@nasa.gov

bNASA Ames Research Center, Moffett Field, CA 94035, USA. E-mail: Richard.Boyle@nasa.gov

Ocular responses to head perturbations consist of intermingled segments termed "slow" (nystagmus) or "fast" (saccade), according to their average speed characteristics. Currently, valuable information that may be contained in saccade data is ignored due to a lack of suitable methodology available to analyze it, and vestibulo-ocular reflex (VOR) health is assessed using inaccurate ad hoc linear methodology or a clinician's ability to judge abnormal eye movements. Here, we develop an a posteriori non-linear tool to quantify VOR health. This goal is accomplished by constructing a diagonal block-oriented data matrix and exploiting its natural sparseness, allowing this novel modeling and analysis technique to achieve these goals concurrently. Our VOR modeling and estimation tool allows for the objective assessment of VOR health by rendering it feasible to analyze both nystagmus and saccade phases simultaneously and efficiently from a single experimental record. This tool provides robust information to the clinician or a trained flight crew enabling an accurate evaluation vestibular health.

A good sense of balance is vital for a pilot/astronaut to safely operate advanced research aircraft or civilian 
passenger aircraft, heavy equipment operators and persons who drive cars or motorcycles etc. This tool may lead to a more timely and comprehensive evaluation for VOR disease quantification and progression possibly providing insights for innovative therapeutic treatment modalities. In addition, the development of a tool for VOR health assessment has important implications not only for public safety, but also for exploratory class missions requiring optimal crew performance.

\section{4-11 [\#3028]}

Gaze control with and without additional retinal optokinetic stimulation on cosmonauts after prolonged spaceflight

L.N. Kornilova ${ }^{\mathrm{a}}$, I.A. Naumov ${ }^{\mathrm{a}}$ and E.V. Drynkina ${ }^{\mathrm{b}}$

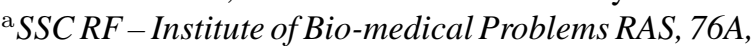
Khoroshevskoe shosse, Moscow, 123007, Russia

${ }^{\mathrm{b}}$ M. V. Lomonosov Moscow State University, GSP-1, Leninskie Gory, Moscow, 119991, Russia

In the ground experiment "SENSORY ADAPTATION" which is a part of the ISS Russian Science Program, we have investigated characteristics of the gaze control (smooth pursuit, saccades, gaze holding) with and without additional retinal optokinetic stimulation (ROKS). The study involved 26 Russian ISScrewmembers before and after a prolonged exposure to weightlessness (126-195 days). Examinations were carried out on L-45 and L-30 preflight and R+1-2, R+45, $\mathrm{R}+8-9$, and $\mathrm{R}+14-19$ postflight. Cosmonauts were required to acquire the foveal stimulus (a small white square with angular displacement less than $1^{\circ}$ ) against the clear background or against the ROKS (a variety of blurred ellipses with angular displacement $\sim 2-4^{\circ}$ ) moving horizontally or vertically. All visual tracking tests were performed using a videooculography recording with a head fixed by a head holder. To evaluate additional spontaneous eye movements we have used a special test with both EOG (eyes closed) and VOG (eyes opened) recording in central and eccentric positions of the eyes. Oculomotor reactions were analyzed using parametric and nonparametric methods of multiple comparisons (ANOVA), correlation and cluster analysis. We have found that visual tracking on a clear background was accompanied by a statistically significant decrease of all characteristics being evaluated (latency, amplitudes, peak velocities, gain, precision etc.) until $\mathrm{R}+8-9$ for all cosmonauts and, for some cosmo- nauts, until $\mathrm{R}+14-19$. However, in the same tests with an additional ROKS even on $\mathrm{R}+4-5$ several cosmonauts had a significant improvement of the gaze control while its characteristics were similar to the preflight, baseline values.

\section{4-12 [\#3043]}

Enhancements of vection in depth from viewpoint oscillation: Effects of field of view, amplitude, focal distance and body posture

\author{
J.E. Zacher ${ }^{\mathrm{a}}$, P.S. Guterman ${ }^{\mathrm{a}}$, S.A. Palmisano ${ }^{\mathrm{b}}$ and \\ R.S. Allison ${ }^{\text {a }}$ \\ ${ }^{a}$ Center for Vision Research, York University, Toronto, \\ Canada \\ ${ }^{\mathrm{b}}$ School of Psychology, University of Wollongong, Wol- \\ longong, Australia
}

Incorporating jitter or oscillation of the vantage point in visual displays produces more compelling illusions of self- motion (vection), despite generating greater sensory conflicts [1]. We are working with the Canadian Space Agency to develop an experiment to study this phenomenon on the International Space Station. Pragmatic issues favour small, near displays rather than typical immersive displays. This paper studies impact of display characteristics on the jitter/oscillation enhancement on vection.

Methods: Visual displays simulated constant velocity forward motion at $1.33 \mathrm{~m} / \mathrm{s}$ through a virtual world, or the same motion with simulated viewpoint oscillation, on a laptop monitor viewed through an aperture. Various experiments examined the effect of oscillation amplitude, direction, field of view (with a different monitor), focal distance and body posture on vection responses.

Results: Adding simulated horizontal or vertical viewpoint oscillation to radial flow increased vection a similar amount. Vection strength was increased more for oscillation peak velocities of $0.28 \mathrm{~m} / \mathrm{s}$ compared to $0.09 \mathrm{~m} / \mathrm{s}$. Increasing focal distance by the use of $+2 \mathrm{D}$ ophthalmic lenses did not measurably impact reported strength of vection. While field of view had no effect, closer viewing distances reduced vection but had no significant effect on the oscillation enhancement.

Discussion: Motion sickness and spatial disorientation continue to impact the availability and effectiveness of astronauts. The current results will guide the development of ISS studies to improve our understanding of how vestibular and visual signals are recalibrated in altered gravity. 


\section{Reference}

[1] S. Palmisano, R.S. Allison and Pekin, Perception 37 (2008), 22-33.

\section{4-13 [\#3054]}

\section{Otolith-ocular responses during counter-rotation in mice}

P. Armstrong ${ }^{\mathrm{a}}$, S. Wood ${ }^{\mathrm{a}, \mathrm{b}}$ and T. Makishima ${ }^{\mathrm{a}}$

${ }^{a}$ Department of Otolaryngology, University of Texas Medical Branch, 301 University Blvd., Galveston, TX 77555-0521, USA

${ }^{\mathrm{b}}$ Universities Space Research Association, NASA Johnson Space Center, Houston, TX 77058, USA

Introduction: In the 5th symposium in this series, Benson and Barnes (1970) described the modulation of horizontal eye movements in human subjects exposed to a rotating linear acceleration vector without angular acceleration (counter-rotation). This linear acceleration stimulus is similar to Off-Vertical Axis Rotation (OVAR), except that during OVAR the semicircular canals are stimulated during the establishment of the acceleration vector. The purpose of this study was to establish baseline ocular responses in mice using a similar counter-rotation paradigm as a test of otolith-ocular function.

Methods: Seven C57BL6 mice between ages 4 to 6 months were restrained onto a counter-rotation platform inside a light-tight drum. Two dimensional eye movements were measured using video-oculography. Mice were tested in both clockwise and counterclockwise directions from $30-180 \%$ s (corresponding to $0.08-0.5 \mathrm{~Hz}$ ).

Results and conclusions: Similar to other otolithocular studies, a frequency dependent horizontal nystagmus was observed that continued throughout rotation in either direction. The largest nystagmus response occurred during rotation $90^{\circ} \%$ s to $150 \%$ s $(0.25$ to $0.4 \mathrm{~Hz}$ ). We conclude that counter-rotation is a viable test of otolith-ocular function in mice. This paradigm will be useful to examine human ortholog genes and proteins necessary for development and maintenance of balance that have been identified and can be easily manipulated in mice.

\section{4-14 [\#3055]}

\section{Sensory re-weighting with changes in vestibular and cutaneous sensitivity following short duration space flight (HYPERSOLE)}

C.R. Lowrey ${ }^{\mathrm{a}}$, S.D. Perry ${ }^{\mathrm{b}}$, N.D. Strzalkowski ${ }^{\mathrm{a}}$, L. Taylor $^{\text {c }}$, S.J. Wood ${ }^{\text {d,e }}$, D.R. Williams ${ }^{\mathrm{f}}$ and L.R. Bent ${ }^{\mathrm{a}}$ ${ }^{a}$ University of Guelph, Guelph, ON, Canada. E-mail: clowrey@uoguelph.ca

${ }^{\mathrm{b}}$ Wilfrid Laurier University, Waterloo, ON, Canada

${ }^{\mathrm{c}}$ WYLE Integrated Science and Engineering, Houston, TX, USA

${ }^{\mathrm{d}}$ NASA Johnson Space Center, Houston, TX, USA

e Universities Space Research Association, Houston, TX, USA

${ }^{\mathrm{f}}$ Faculty of Health Sciences, McMaster University, Hamilton, ON, Canada

Information from the vestibular system is coupled with somatosensory input from the feet and lower limbs to facilitate orientation of the body in the environment [1]. Anecdotal evidence suggests that the skin on the foot sole may become hypersensitive during and following spaceflight. The extent of skin hypersensitivity and its impact on postural disequilibrium observed following space flight have not been documented to date.

To assess skin contributions, four vibration frequencies $(3,25,60$, and $250 \mathrm{~Hz})$ were used to assess the vibration sensitivity of the four classes of mechanoreceptors at three sites on the foot sole (great toe, 5th metatarsal head, heel). Nylon monofilaments (0.026 to $110 \mathrm{~g}$ of force) applied normal to the surface of the skin assessed static sensitivity thresholds. These data were coupled with functional balance tests (computerized dynamic posturography, CDP) to relate skin hypersensitivity with vestibular changes and balance function.

We hypothesize that skin sensitivity will increase Post-Flight and will correlate to changes in balance control. Preliminary data analyses from three subjects suggest that skin sensitivity is selectively increased at different sites, with greater incidence of hypersensitivity at the 5 th metatarsal head. Increased sensation from skin may be due to sensory re-weighting with an altered gravito-inertial environment [1]. Specifically, as vestibular inputs are reduced information from the skin is increased. Insight into balance challenges as a result of heightened skin input will enhance current theories on skin contribution to postural control, and may be applied to long duration spaceflight in the future. 


\section{Reference}

[1] B.L. Day et al., Adv Exp Med Biol 508 (2002), 129-137.

\section{4-15 [\#3058]}

\section{New method for quantifying human high-frequency linear and rotational VOR during launch- relevant conditions}

D.B. Liston ${ }^{\mathrm{a}, \mathrm{b}}$, B.D. Adelstein ${ }^{\mathrm{a}}$, B.R. Beutter ${ }^{\mathrm{a}}$ and L.S. Stone $^{\mathrm{a}}$

${ }^{a}$ NASA Ames Research Center, Moffett Field, CA, USA

${ }^{\mathrm{b}}$ San Jose State University, San Jose, CA, USA

The human vestibular system stabilizes one's conjugate gaze point in 3D in the face of self-motion disturbances with a 6 degrees-of-freedom VestibuloOcular Reflex (VOR). We have constructed a videobased system that can track the stabilizing binocular smooth eye movement response to the linear and rotational vestibular perturbations that would typically occur during launch (chest-to-spine vibration during into-the-chest sustained $\mathrm{G}$ loads of $1 \mathrm{~g}$ or higher). Our new method enables the precise measurement ( $4 \mathrm{~ms}$ and $0.01^{\circ}$ or better resolution) of a gaze-stabilization response dominated by the combined pitch (rotation) and heave (linear) VORs at frequencies in the 5 to $25 \mathrm{~Hz}$ range and for vibration levels of up to $\pm 1 \mathrm{~g}$. To validate this method, for self-generated rotational pitch motion of about $\pm 5^{\circ}$ at about $0.7 \mathrm{~Hz}, 1$ ) we measured the gaze angle (A, solid gray line) by tracking the image of the pupils and subtracting out head motion, 2) we measured the head position by tracking the image of a fiducial triangle, and 3) we computed the headinduced demand (A, dashed black line) for oculomotor compensation by adding the arctangent of heave over viewing distance (linear demand) with the pitch angle change (rotational demand). The power density curves (B) for oculomotor demand and gaze angle nearly superimpose as expected for near perfect VOR compensation. Our plan is to use this new approach to document the trade-space between VOR gain and Gload-plus-vibration condition to determine when gaze stabilization becomes inadequate to support effective human performance in spaceflight-relevant tasks (i.e., reaching, dynamic visual acuity, pursuit, visual target acquisition). The data will guide future spacecraft interface design and operational concepts, independent of the specific launch conditions.
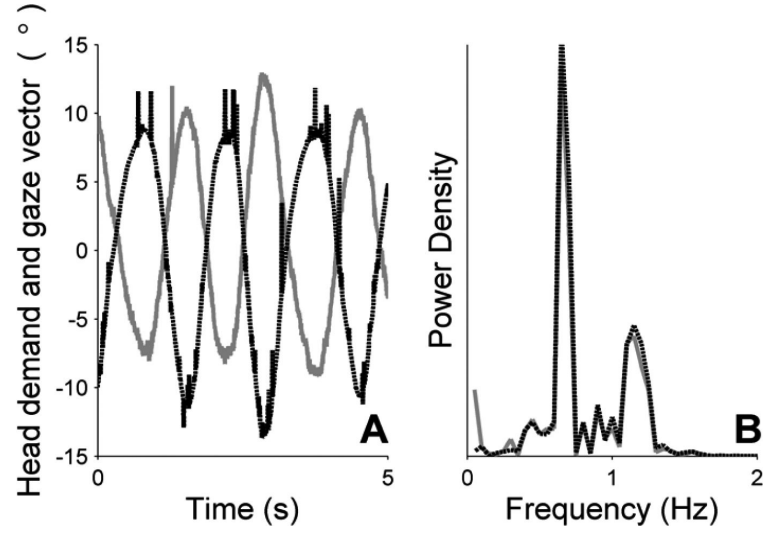

\section{4-16 [\#3002]}

\section{Motion sickness etiology: A cholinomimetic agent} hypothesis

S. Sheehan ${ }^{\mathrm{a}}$, C. Oman ${ }^{\mathrm{b}}$ and K. Duda ${ }^{\mathrm{b}}$

a Brigham and Women's Hospital, Department of Radiology, 75 Francis St., Boston, MA 02115, USA. E-mail: sesheehan@partners.org

${ }^{\mathrm{b}}$ Man Vehicle Lab, Department of Aeronautics and Astronautics, MIT, Cambridge, MA 02139-4307, USA. E-mail:coman@mit.edu

Motion sickness has been defined as a set of physiological signs and symptoms produced as a result of prolonged sensory conflict in central nervous system vestibular centers. It has long been noted that the particular pattern of motion sickness signs and symptoms does not fit the conventional "fight or flight vs. rest and digest" autonomic synergy. We argue that most of the progression of symptoms is consistent with a new etiologic hypothesis: that an as-yet-unidentified ganglionic cholinomimetic agent is slowly released in proportion to sensory conflict. The agent accumulates systemically and stimulates the peripheral sympathetic and parasympathetic ganglia, the adrenal medulla, and potentiates the response of central cholinergic emetic pathways to the same conflict stimulus. The predominant effects of ganglionic stimulation on each autonomic organ, determined by resting tone, are selectively enhanced or inhibited by adrenal stress hormone release, producing the atypical pattern of autonomic changes seen in motion sickness. The adrenergic response may eventually also counter the central emetic drive. The hypothesis could be experimentally pursued via human and animal experiments employing a nonselective cholinergic antagonist that has both central and peripheral ganglionic actions such as mecamylamine. 
Acknowledgement: C. Oman was supported by the National Space Biomedical Research Institute through NASA NCC9-58. S. Sheehan was supported by the department of Diagnostic Radiology at the Brigham and Women's Hospital, Boston, MA, USA.

\section{4-17 [\#3009]}

\section{Visual bias predicts gait adaptability in novel sensory discordant conditions}

R.A. Brady ${ }^{\mathrm{a}}$, C.D. Batson ${ }^{\mathrm{b}}$, B.T. Peters ${ }^{\mathrm{a}}$, A.P. Mulavara $^{\mathrm{c}}$ and J.J. Bloomberg ${ }^{\mathrm{d}}$

${ }^{\mathrm{a}}$ Wyle Integrated Science and Engineering Group, Houston, TX, USA

${ }^{\mathrm{b}}$ MEI Technologies, Inc., Houston, TX, USA

${ }^{\mathrm{c}}$ Universities Space Research Association, Houston, TX, USA

${ }^{\mathrm{d}}$ Neuroscience Laboratories, NASA Johnson Space Center, Houston, TX, USA

We designed a gait training study that presented combinations of visual flow and support-surface manipulations to investigate the response of healthy adults to novel discordant sensorimotor conditions. We aimed to determine whether a relationship existed between subjects' visual dependence and their postural stability and cognitive performance in a new discordant environment presented at the conclusion of training (Transfer Test). Our training system comprised a treadmill placed on a motion base facing a virtual visual scene that provided a variety of sensory challenges. Ten healthy adults completed 3 training sessions during which they walked on a treadmill at $1.1 \mathrm{~m} / \mathrm{s}$ while receiving discordant support-surface and visual manipulations. At the first visit, in an analysis of normalized torso translation measured in a scene-movement-only condition, 3 of 10 subjects were classified as visually dependent. During the Transfer Test, all participants received a 2-minute novel exposure. In a combined measure of stride frequency and reaction time, the non-visually dependent subjects showed improved adaptation on the Transfer Test compared to their visually dependent counterparts. This finding suggests that individual differences in the ability to adapt to new sensorimotor conditions may be explained by individuals' innate sensory biases. An accurate preflight assessment of crewmembers' biases for visual dependence could be used to predict their propensit ies to adapt to novel sensory conditions. It may also facilitate the development of customized training regimens that could expedite adaptation to alternate gravitational environments.

Acknowledgement: This work was supported by the National Space Biomedical Research Institute through NASA NCC 9-58. 